\title{
A Cell Centered Database (CCDB) for Multi-Scale Microscopy Data Management
}

\author{
S. Zhang, ${ }^{*}$ D. Price, ${ }^{*}$ X. Qian, ${ }^{* *}$ A. Gupta, ${ }^{* *}$ M. Wong, ${ }^{*}$ M.H. Ellisman,, ${ }^{* * *}$ and M.E. Martone ${ }^{*, *}$ \\ * National Center for Microscopy and Imaging Research, Center for Research in Biological Structure \\ and Dept. of Neurosciences, University of California, San Diego, La Jolla, CA 92093-0608 \\ ** San Diego Supercomputer Center, University of California, San Diego, CA 92093-0505, USA
}

We have developed an on-line database (http://ncmir.ucsd.edu/CCDB) for multi-resolution 3D structural and protein localization imaging data from light and electron microscopy, including correlated microscopy. The Cell Centered Database (CCDB) was developed using Oracle and models the entire process of $3 \mathrm{D}$ reconstruction, from experiment to segmentation. The CCDB was created as a resource for microscopists, structural biologists and computer scientists interested in 3D biological imaging. It is currently populated with data obtained at the National Center for Microscopy and Imaging Research, but is available to outside users. Additional description of the purpose and the types of data can be found in Martone et al. [1]. The CCDB was designed to be extensible so that additional types of imaging data can be added as they become available.

The CCDB provides comprehensive descriptive, structural, and administrative information on microscopy datasets and 3D reconstructions categorized based on reconstruction technique. To accommodate the many types of microscopy data that are obtained by researchers, we created the concept of the "microscopy product." The microscopy product refers to a related set of 2D images derived from either light or electron microscopy, e.g., time series, optical section series, serial sections, montages or tilt series. These image sets may be used to create 3D reconstructions, animations or segmented objects. A single record in CCDB comprises a microscopy product along with any derived 2D images, 3D reconstructions, animations, segmented objects or measurements. Pointers to correlated data sets are also included. There are currently three security levels for data in CCDB: 1) private; 2) group; and 3) public. Each reconstructed image is tagged with ownership and a security level. If a dataset is made available to the public, users may view descriptive and quantitative data and download original imaging data. If a data set is marked for private or group access, users are able to view a limited set of descriptive information and low resolution thumbnails, but only the user (or group) who has the ownership of particular reconstructed image is able to retrieve the actual images. Permission to download the original data may be obtained from the owner via e-mail. The CCDB also contains information on obtaining visualization and analysis tools for viewing and manipulating the imaging data.

An overview of the CCDB website is shown in FIG. 1. Users must obtain an account but the CCDB is available free of charge. A user can issue queries based on image metadata or/and segmented object attributes in simple query form or customized advanced query form. Additional query forms are currently under development and will include the ability to issue statistical queries. A typical query result is shown in FIG. 2. The query result page displays summary information on the image datasets, and a thumbnail picture of each individual raw image, reconstructed image, segmented image. In addition, the brief result has links to: 1) Download the original image; 2) Display the measurements of the segmented object; and 3) Display correlated light or electron microscopy image dataset etc. The CCDB provides a knowledge exploration and new discovery environment for 
researchers to access raw image data, volume reconstructions and segmented products from light and electron microscopic studies on cell structure and macromolecular distribution.

\section{References}

[1] M.E. Martone et al., Journal of Structural Biology, 138 (2002) 145.

[2] This research was sponsored by NIH grants from NCRR RR04050, RR08605 and the Human Brain Project DC03192, and NSF grants supporting the National Partnership for Advanced Computational Infrastructure NSF-ASC 97-5249 and MCB-9728338.

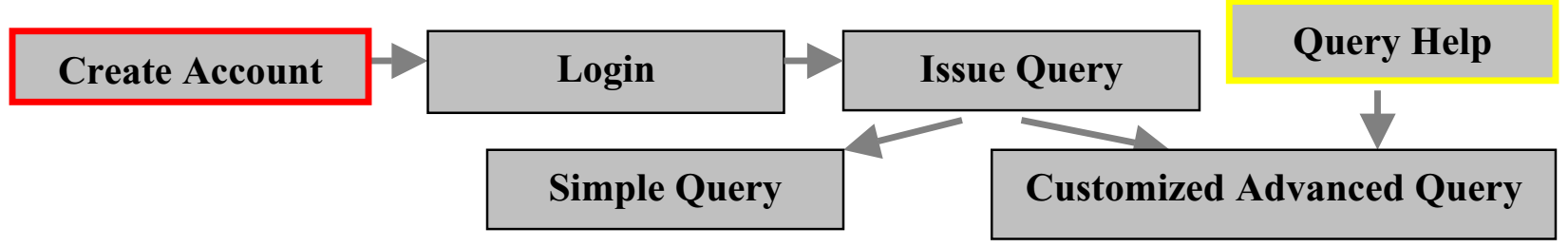

FIG. 1: A road map of the CCDB web site.

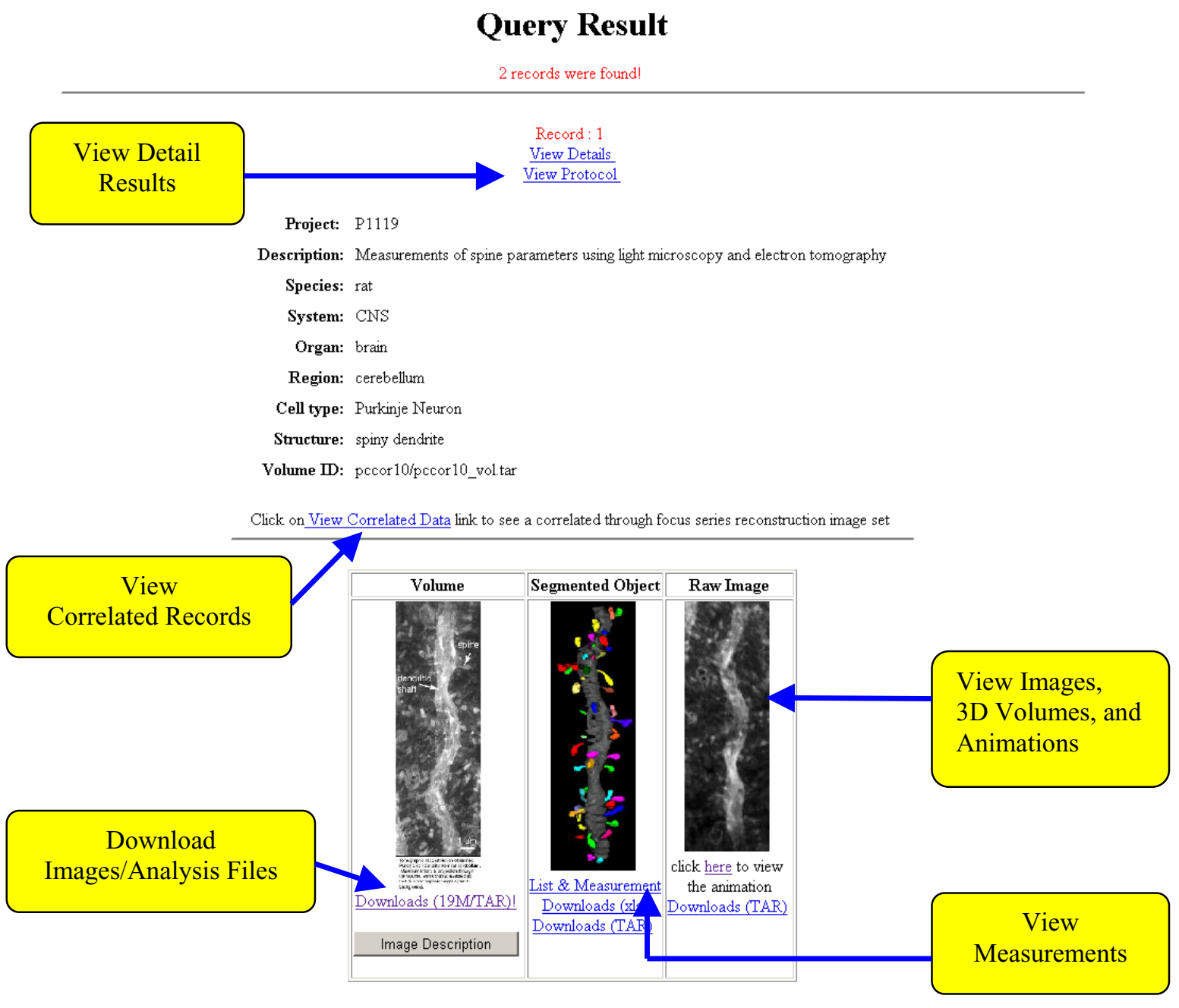

FIG. 2. A typical query result page. 